\title{
Simultaneous optimization of electrical interconnection configuration and cable sizing in offshore wind farms
}

\author{
Mohsen SEDIGHI ${ }^{1,2}$, Mohammad MORADZADEH ${ }^{3}$, \\ Osman KUKRER ${ }^{1}$, Murat FAHRIOGLU ${ }^{4}$
}

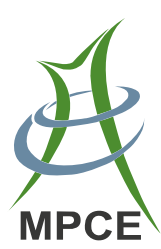

\begin{abstract}
Offshore wind farm (OWF) is the largest renewable energy resource. The electrical interconnection cost of OWFs is a considerable fraction of the overall design cost of the farm. In order to minimize the investment and operational costs, this paper proposes an optimization formulation to find the optimal electrical interconnection configuration of wind turbines (WTs), and the optimal cable sizing simultaneously. This simultaneous minimization of total trenching length and cable dimensions creates a complex optimization problem that is solved by the harmony search (HS) algorithm. In this paper, two distinct methods of full and partial optimal cable sizing are
\end{abstract}

CrossCheck date: 7 November 2017

Received: 24 May 2017/Accepted: 7 November 2017/Published online: 18 January 2018

(C) The Author(s) 2018. This article is an open access publication

Mohsen SEDIGHI

mohsen.sedighi@cc.emu.edu.tr

Mohammad MORADZADEH

m.moradzadeh@srttu.edu

Osman KUKRER

osman.kukrer@emu.edu.tr

Murat FAHRIOGLU

fmurat@metu.edu.tr

1 Department of Electrical and Electronic Engineering, Eastern Mediterranean University (EMU), Gazi Magusa,

North Cyprus, Mersin 10, Turkey

2 Electrical Engineering Department, Sari Branch, Islamic Azad University (IAU), Sari, Iran

3 Electrical Engineering Department, Shahid Rajaee Teacher Training University, Lavizan, Tehran, Iran

4 Department of Electrical and Electronics Engineering, Middle East Technical University-Northern Cyprus Campus, Güzelyurt, Mersin 10, Turkey considered to comprehensively assess the optimal interconnection layout of OWFs. Furthermore, various shipping and burying costs as well as various WTs power ratings are considered in order to investigate their impact on the optimal electrical interconnection system. The optimal electrical interconnection design obtained by the HS algorithm corresponds to a lower cost that together with the technological developments can help policy makers increase the use of offshore wind energy as a feasible unlimited renewable resource in their energy production portfolios.

Keywords Offshore wind farm (OWF), Optimal interconnection configuration, Cable sizing, Simultaneous optimization, Harmony search (HS) algorithm

\section{Introduction}

Wind energy is the world's fastest growing renewable energy resource. Offshore wind farms (OWFs) can play a significant role in reducing the environmental implications of meeting the high electrical power demand of modern societies. The EU policy framework for securing $27 \%$ renewable energy and $40 \%$ reduction in greenhouse gas emissions could be met with the OWFs solution with expected growth to $65 \mathrm{GW}$ by 2030 [1]. In fact, wind speed is higher and more persistent in offshore areas, thus wind turbines (WTs) can be designed for higher speed ratio and smaller weight for the same power rating. Land availability and acoustic emission, which are significant issues in onshore wind farm design, are insignificant in the OWF design. However, the foundations, installation and maintenance costs, as well as cost of the electrical interconnection system and transmission line to the shore are 
significantly higher in OWFs [2]. These higher costs have demanded extensive technical developments and optimization challenges in order to reduce the levelized cost of energy (LCoE). It is estimated that $\mathrm{LCoE}$ could be reduced to $100 \$ / \mathrm{MWh}$ by 2030 [1].

Conventionally, the electrical interconnection system of OWFs could be structured in different configurations such as radial, star or loop design depending on the desired reliability level. Nevertheless, probability of a fault in buried subsea (submarine) cable is very low, about 0.001/ $\mathrm{km} / \mathrm{year}$ [3]. In the last few decades, most of the installed OWFs have had relatively small area or utilized WTs with low power rating $(\leq 4 \mathrm{MW})$. Hence, finding optimal interconnection configuration was not necessary and could be structured with a simple electrical design such as typical radial configuration.

Since large-scale OWFs have been developed in the last decade, more research works have recently conducted on the optimization of various aspect of OWF. Some studies only found the optimal electrical interconnection configuration to minimize the total trenching length by utilizing analytical methods. Reference [4] addressed different typical power collecting systems and proposed a new single return loop design to improve the reliability and minimize the cables cost. Dutta and Overbye [5] proposed a clustering-based cable design, different from the typical configurations. In a later study [6], the authors proposed various clustering methods to decrease the problem size and find the optimal configuration of each cluster by the minimum spanning tree (MST) algorithm. This study additionally proposed an algorithm to introduce intermediate splice point to minimize the total trenching length of on-shore wind farms. Similar clustering methodology based on the MST algorithm [7, 8] were proposed for OWFs by ignoring intermediate splice point due to very high splicing costs of on subsea cables. Banzo and Ramos [9] proposed a stochastic programming model to find the optimal layout of the electric power system of a real OWF in the UK. A mixed integer programing (MIP) algorithm was used and a minor cost reduction was obtained. Lumbreras and Ramos [10] proposed a similar tool with reasonable computational time to find the optimal electrical layout of OWFs by the MIP algorithm and decomposition strategies. A mixed integer linear programming (MILP) approach was utilized in [11] to minimize the total trenching length of OWFs to find optimal location of offshore substation (OS) and optimal interconnection configuration individually. Nevertheless, none of above papers could include cable dimensions in the objective function to represent a realistic optimization, due to utilizing MST or analytical optimization methods that at least require constant weights for all edges. Reference [12] proposed an improved genetic algorithm (GA) to find optimal location of WTs to minimize the wake effect and then the total trenching length without cable sizing to find optimal electrical interconnection configuration of an OWF in two individual steps. In addition, crossing subsea cables was not avoided, which may increase burying costs or difficulty in practice. On the contrary, recent large-scale OWFs utilizing WTs with higher power rating ( $\geq 5 \mathrm{MW}$ ) have created a new challenge to optimize the electrical interconnection layout (interconnection configuration and cable sizing) of the OWFs. Recently, variable cable dimension has also been considered to find the optimal electrical interconnection configuration of OWFs, where metaheuristic algorithms were utilized. Reference [13] proposed a modified approach to the traveling salesman problem (TSP) for designing a radial configuration. This study used an improved GA to find the optimal electrical network design of a large-scale OWF, while cable dimensions were considered as a radial configuration without any tapering for cables away from the OS. A binary GA was used in [14] to find the optimal electrical network of an OWF where full cable sizing was considered. However, the optimal layout was found just for the pre-clustered nodes in order to decrease the problem size and power loss as an operational cost is not considered. Hou et al. in [15] proposed an adaptive particle swarm optimization (APSO), which adjusts every individual solution to new integer solutions to solve discrete problems. In order to minimize the total cost of cables, an APSO-MST algorithm was utilized to find the optimal interconnection layout and OS location of an OWF simultaneously. However, the total power losses as well as shipping and burying costs $\left(C_{\mathrm{sb}}\right)$ of subsea cables were not considered. In [16], the authors also found the optimal interconnection and transmission system of an OWF, where APSO-MST algorithm and C-means clustering method were utilized. In their recent studies [17], the optimal location of OS and optimal topology of WTs location were simultaneously found together with the optimal interconnection layout of real OWFs. The same APSO-MST algorithm was adopted to minimize wake effect and cable cost of the OWF, due to high performance of PSO to solve such continuous problems (finding the optimal locations). However, the project area of the OWFs in case study was considered increasable during the optimization process, which would not yield to fair results. On the contrary, in these studies, finding the optimal electrical interconnection configuration and cable sizing of an OWF are inherently discrete problems. Thereby, the APSO, by adjusting real values to integers, may not be the best choice, as its performance was decreased to solve such a complex problem. Furthermore, similar [14], crossing subsea cables was avoided so that no crossed edges were considered in MST, which may miss some feasible solutions. 
The existing optimization methods or formulations in the literature do not seem to be completely satisfactory. This is because, finding the optimal electrical interconnection layout without cable sizing leads to over-dimensioning of the interconnecting cable system. Moreover, decreasing the problem size by any clustering method without considering integration of all WTs or utilizing an insufficient optimization algorithm for such a complex problem may result in a local optimal solution.

In this study, the optimal interconnection configuration and cable sizing of an OWF are found simultaneously to minimize the capital expenditure (CAPEX) of the AC electrical interconnection system. The main contributions of this paper are as follows:

1) Formulation of the OWF interconnection system with two different cable sizing methods (full and partial), offering two distinct solutions according to the seabed conditions or cable installation constraints.

2) Investigation of the impact of the WTs' power ratings and $C_{\mathrm{sb}}$ on the optimal layout of electrical interconnection system.

3) Detection and avoidance of cable crossing.

4) Use of the harmony search (HS) as a high performance algorithm to solve the formulated discrete optimization problem, without the need for any clustering method.

5) Provision of a useful tool to find the optimal interconnection layout of a large-scale OWF with any given topology, with possible adoption to on-shore wind farms.

The remainder of the paper is organized as follows. The computational complexity of this problem is investigated in Section 2. In Section 3, the HS algorithm is described. Section 4 discusses about formulating the optimization problem. The case study is introduced in Section 5. In the next section, the results are illustrated and investigated in three distinct scenarios. Finally, Section 7 summarizes the conclusions.

\section{Computational complexity of the problem}

The simultaneous optimal electrical interconnection configuration and cable sizing of an OWF as a discrete optimization problem is rather similar, but harder than both the traveling salesman problem (TSP) and optimizing pipe diameters in the water distribution system (WDS) problem. It is harder, because the traveling cost of each individual road (branch) in the TSP is constant, and the network configuration in the WDS problem is given according to urban design restrictions. In fact, simultaneous optimal pipe diameters design and configuration of system network in a WDS problem have never been optimized. This simultaneous optimization problem was defined as an extremely complex optimization problem [18]. Note that, these two well-known discrete optimization problems are also non-smooth and non-convex problems. Thereby, they are defined as NP-complete problems [19, 20]. However, finding the simultaneous optimal configuration and cable sizing of an OWF without any clustering method can be embedded in the category of NP-hard or at least NPcomplete problem [13]. Because, including corresponding cost of cable dimensions into the objective function causes variable cost branches (edges) for each individual solution, thus creating an extremely complex and inherently intractable discrete optimization problem. Generally, these optimization problems can be regarded as a class of complex combinatorial problems that cannot be mathematically modeled, and be solve with analytical optimization algorithms or they may result in a local optimal solution. Therefore, metaheuristic optimization techniques would be required to solve such complex problems.

On the one hand, the complexity and the number of possible solutions for the optimal electrical interconnection configuration problem generally increases dramatically with factorial factor of the number of optimization variables. However, by an intuitive engineering insight, it can easily be understood that most of the possible power flow directions cannot comprise an optimal power flow path. Hence, a generalized sub-algorithm has been developed to reduce the search space of a given topology OWF, according to the location coordinates of WTs and OS with respect to each other (either symmetric or asymmetric topology of OWF). The proposed reduction sub-algorithm identifies only the feasible connecting nodes (either WTs or OS) to each individual WT with acceptable paths to the OS location. Nevertheless, any unlikely but feasible connecting nodes have been considered in the decision set. Only the paths between WTs in opposite of OS direction are not included into the decision set. Thereby, the number of feasible solutions for interconnection configuration problem can be reduced to exponential-time problem. On the other hand, adding cable sizing to the problem increases the problem complexity by an exponential-time factor. Number of possible solutions for the cable sizing problem is $m^{N}$, where $m$ is limited to the number of available cable dimensions (for example only three choices in partial cable sizing). The ampacity and required cable dimension of each individual branch can easily be determined after finding the interconnection configuration of each individual solution. 


\section{HS algorithm}

Metaheuristic optimization algorithms mimic natural phenomena. They include biological evolution in the GA, physical annealing in the simulated annealing (SA), human/animal memory in the tabu search (TS) and particle swarm optimization (PSO). Reference [21] conceptualized HS algorithm as another artificial phenomenon from the musical process of searching for a perfect state of harmony such as jazz improvisation.

The HS algorithm is a new metaheuristic optimization method and has been used to tackle various optimization problems in discrete and continuous space successfully [22]. The capability of solving complex and discrete optimization problems was demonstrated by finding the most proper solution in TSP and WDS in comparison with GA and other analytical optimization methods [20, 21, 23].

The steps in the HS algorithm procedure are as follows:

Step 1 Initialize a harmony memory (HM).

Step 2 Improvise a new harmony from $\mathrm{HM}$, or at random.

Step 3 If the new harmony is better than the worst harmony in HM, include the new harmony in HM, and exclude the worst harmony from HM.

Step 4 If stopping criteria are not satisfied, go to Step 2.

The parameters of the HS algorithm are the harmony memory size (HMS), harmony memory considering rate (HMCR) and pitch adjusting rate (PAR). The HMCR and PAR parameters help the HS algorithm to find improved solutions globally and locally, respectively. Recommended ranges for parameter values are 0.70-0.95 for HMCR and 0.05-0.70 for PAR, according to the frequently used values in other HS applications. Besides, according to the number of optimization variables, HMS value of $10-100$ is recommended [23].

In this paper, HMS and the maximum number of iterations are assumed as $N$ and $2 N \times 10^{3}$, where $N$ is the number of discrete variables in this optimization. A variable HMCR has been used where it increases with the rate of 0.0001 from 0.7 to 0.95 per iteration. However, no extra pitch adjustment has been used in this optimization problem, since it is an arbitrary operation and this adjustment is experimentally helpful just in local search of continuous problems.

A HM with HMS of four, shown in Fig. 1, is a memory location where all the solution vectors and their corresponding fitness values are stored [21]. This HM is similar to the genetic pool in the GA. Consider a jazz trio composed of fiddle, saxophone and keyboard (three optimization variables). Initially, the memory is stuffed with four random harmony vectors: $(C, E, G),(B, D, A),(C, F, A)$,

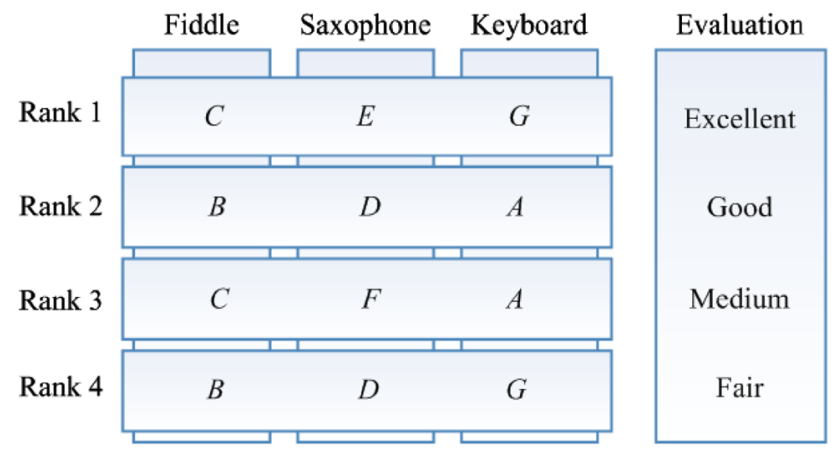

Fig. 1 Structure of harmony memory

and $(B, D, G)$ that are sorted with their corresponding aesthetic estimation.

Improvising procedure of a new harmony for each note is based on the following three rules [23]:

1) HM consideration with probability of HMCR.

2) Extra pitch adjustment with probability of PAR.

3) Random selection with probability of (1-HMCR).

Every note in HM has the same opportunity to be selected. In the improvising procedure, three instruments produce a new harmony; for example $(C, D, A)$.

If evaluation of the new harmony vector is better than the worst harmony (4th rank) in HM, then the new harmony is included into the HM and the worst harmony is excluded from the HM. After ranking the HM again, this process is repeated until satisfying results according to the stopping criteria are obtained [21,23].

\section{Optimization problem}

In this study, the simultaneous optimal electrical interconnection configuration and cable sizing of OWFs minimizes the trenching length and cable dimensions of a given topology. In order to minimize the investment and operational costs, the proposed optimization formulation considers both cable cost as well as the sum of shipping and burying costs $\left(C_{\mathrm{sb}}\right)$, as a significant influencing term in this optimization problem.

$C_{\mathrm{sb}}$ varies greatly with respect to several environmental and practical parameters in each individual OWF (e.g. seabed geology, distance to shore, burial depth, the OWF size, etc.). In this study, if practical detailed costs are provided, even a variable $C_{\mathrm{sb}}$ can be applied, i.e. different value of variable $C_{\mathrm{sb}}$ can be considered for each individual choice (according to its corresponding seabed condition) of the discrete decision set $\left(B_{i}\right)$. However, similar to all recent studies in the literature a constant value for $C_{\mathrm{sb}}$ has conventionally been considered. In different references, an extensive range of $150-1000 \$ / \mathrm{m}$ is assigned for $C_{\mathrm{sb}}$ of 
medium voltage (MV) cables [14, 24]. In case of adopting the proposed formulation in on-shore wind farms, laying cost itself with other additional limitation (e.g. land unavailability or other practical restrictions) can be considered as a variable cable installation cost instead of a constant cost for each individual choice of the discrete decision set $\left(B_{i}\right)$.

Due to the significant influence of $C_{\mathrm{sb}}$, two methods of cable sizing are proposed as distinct scenarios. The methods are defined as follows:

1) Full cable sizing: the cable dimension of each branch is assigned individually according to its ampacity.

2) Partial cable sizing: limited number of cable dimension is considered to be available in the decision set (three available dimensions in this study). Moreover, cable dimensions of all branches on each route are identically assigned according to the ampacity of last branch before joining another route or connecting to the OS.

Note that, due to the existence of MV switchgears and junction terminals (at least three cables) below every WT, cutting the subsea cable is inevitable in electrical interconnection of wind farms, thus connecting different cable dimensions in full cable sizing method can be possible. However, cable dimension changing or utilizing more choices in the decision set of cable dimensions may increase $C_{\mathrm{sb}}$ practically. Thereby, a method of partial cable sizing is also proposed in this paper.

\subsection{Mathematical formulation}

For integration of all WTs, during the evolutionary optimization process the WTs could be clustered randomly according to the maximum ampacity of the largest cable dimension, with different shapes and sizes in any iteration. Note that, no specific clustering method is applied before solving the optimization problem. Thereby, in this complex optimization problem, only a small difference may occur between fitness values of the global optimal and local solutions, where it may change the shape and size of clusters as well as the corresponding cable dimension of several paths. Therefore, escaping from local optimum solutions and finding the global optimal solution would be very difficult in a large problem, even with metaheuristic optimization algorithms. In fact, including the cable costs into the objective function creates variable cost branches for each individual solution, thus a complex, non-convex and non-linear optimization problem is formed. Hence, before running the optimization algorithm, the reduction sub-algorithm identifies the discrete decision set $\left(B_{i}\right)$ of the interconnection configuration problem for each individual WT $\left(i^{\text {th }}\right)$. The sub-algorithm can identify $B_{i}$ either for symmetric or asymmetric topology of the OWF, while the location coordinates of all WTs and OS are considered as given input data. After that, the OS location coordinates is considered to be set at the origin. In addition, every WT in its decision set has a chance to be connected to the OS directly, while $B_{i}$ consists of $k_{i}$ feasible choices that are found as follows:

$$
\left\{\begin{array}{cl}
B_{i}=\sum_{n=1, n \neq i}^{N+1} \delta_{n} n & \forall i \in\{1,2, \ldots, N\} \\
\text { s.t. } \sum_{n=1}^{N+1} \delta_{n}=k_{i} & \forall \delta_{n} \in\{0,1\}
\end{array}\right.
$$

where $i$ is WT (optimization variable) index; $n$ is the node (either WTs or OS) index; $N$ is the number of WTs; $k_{i}$ is the number of choices in the decision set for $i^{\text {th }}$ WT; $N+1$ represents the OS index; $\delta_{n}$ is a binary variable $[0,1]$ that is assigned 1 if $n^{\text {th }}$ node is a feasible choice according to the reduction sub-algorithm to be selected in the decision set, otherwise 0 . Since the horizontal spacing of WTs are considered greater than vertical spacing, $\delta_{n}$ in the reduction sub-algorithm in this study is assigned as follows:

$\delta_{n}= \begin{cases}1 & \left|\operatorname{Re}\left(l o c_{i}\right)\right|>\left|\operatorname{Re}\left(l o c_{n}\right)\right| \text { or } l o c_{n}=l o c_{N+1} \\ 0 & \text { otherwise }\end{cases}$

where $l o c_{i}$ and $l o c_{n}$ are Cartesian location coordinates of $i^{\text {th }}$ WT and its $n^{\text {th }}$ feasible choice, respectively $\left(\operatorname{loc}_{i} \in \mathbb{C}\right)$.

The optimization variable of the electrical interconnection problem $\left(y_{i}\right)$ is the index of the next connected node to $i^{\text {th }}$ WT. The value of this optimization variable can be chosen among the discrete decision set of $i^{\text {th }}$ WT $\left(B_{i}\right)$ with $k_{i}$ choices.

$$
\begin{cases}y_{i}=\sum_{x=1}^{k_{i}} \beta_{x} B_{i}(x) & \forall B_{i} \subseteq\{1,2, \ldots, N, N+1\}, i \notin B_{i} \\ \text { s.t. } \sum_{x=1}^{k_{i}} \beta_{x}=1 & \forall \beta_{x} \in\{0,1\}\end{cases}
$$

where $x$ is the choice index of the decision set; $\beta_{x}$ is a binary variable $[0,1]$ that is assigned 1 , if $x^{\text {th }}$ choice of the decision set is chosen randomly, otherwise 0 .

In order to represent an iterative output solution for the interconnection configuration problem an $\mathrm{N}$-length vector (Y) has been defined as (4) for all WTs.

$\boldsymbol{Y}=\left\{y_{1}, y_{2}, \ldots, y_{N}\right\} \quad y_{i} \neq i$

Since a fixed voltage is assumed for all nodes, no power flow analysis is required for the interconnection system. Thereby, after finding the interconnection configuration and assigning $y_{i}$, the ampacity of each branch $\left(I_{i, y_{i}}\right)$ between $i^{\text {th }}$ WT and $y_{i}^{\text {th }}$ node is just determined analytically by the Network Flow method, according to the injected current of each WT $\left(I_{G}\right)$ : 


$$
\begin{cases}I_{i, y_{i}}-\sum_{j=1}^{N} \sigma_{j, i} . I_{j, i}=I_{G} & \forall i, j \in\{1,2, \ldots, N\} \\ \text { s.t. } I_{i, y_{i}}>0 & \forall y_{i} \in\{1,2, \ldots, N, N+1\}, y_{i} \neq i\end{cases}
$$

where $I_{G}$ is the injected current by each WT; $I_{j, i}$ is the ampacity of connected branch from former WT $\left(j^{\text {th }}\right)$ to $i^{\text {th }}$; $\sigma_{j, i}$ is a binary variable $[0,1]$ that represents the branch connection between $i^{\text {th }}$ and $j^{\text {th }}$ WTs. $\sigma_{j, i}$ is assigned 1 if such a connection exist, otherwise 0 .

$\sigma_{j, i}= \begin{cases}1 & y_{j}=i \\ 0 & y_{j} \neq i\end{cases}$

In the full cable sizing method, the assigned cable dimension $\left(c s_{i}\right)$ of each branch is found individually with respect to its ampacity $\left(I_{i, y_{i}}\right)$ for each particular configuration. In the partial cable sizing method, the number of cable dimensions in the decision set is limited (three available dimensions in this paper) and the cable dimensions of all branches of each route are assigned according to the ampacity of last branch before joining another route or connecting to the OS for each particular configuration.

$$
\begin{cases}c s_{i}=\sum_{d=1}^{m} \lambda_{i}\left(a_{d}\right) a_{d} & \forall c s_{i}, a_{d} \in A \\ \text { s.t. } \sum_{d=1}^{m} \lambda_{i}\left(a_{d}\right)=1 & \forall \lambda_{i} \in\{0,1\}\end{cases}
$$

where $d$ is the element index of the set of available cable dimensions $(A) ; m$ is the number of available dimensions in the set of subsea cables; $a_{d}$ is the dimension of $d^{\text {th }}$ element; $c s_{i}$ is the assigned cable dimension for $i^{\text {th }} \mathrm{WT} ; \lambda_{i}\left(a_{d}\right)$ is a binary variable $[0,1]$ of $i^{\text {th }} \mathrm{WT}$ that represents $d^{\text {th }}$ cable dimension index, assigned 1 as defined in (7), otherwise 0 .

$\lambda_{i}\left(a_{d}\right)= \begin{cases}1 & I_{d-1}^{\max }<I_{i, y_{i}} \leq I_{d}^{\max } \\ 0 & \text { otherwise }\end{cases}$

where $I_{d}^{\max }$ is the admissible ampacity of $d^{\text {th }}$ index of subsea cable set. In order to represent an output solution for the cable sizing problem, a second $N$-length vector $(\boldsymbol{C S})$ has been defined as follows:

$\boldsymbol{C S}=\left\{c s_{1}, c s_{2}, \ldots, c s_{N}\right\}$

Table 1 shows the output vectors ( $\boldsymbol{Y}$ and $\boldsymbol{C S}$ ) of an individual solution corresponding to the optimal solution of the first case study of this paper. Due to space limitations, instead of all 63 WTs, only the output vectors of the first eleven WTs are depicted.

Figure 2 provides a flowchart of the HS (or GA) optimization algorithm. Three penalty terms are applied in this optimization process (cable crossing, looping and
Table 1 Part of output vector of individual solution

\begin{tabular}{lrrlrllllrrr}
\hline $\operatorname{Index}(i)$ & 1 & 2 & 3 & 4 & 5 & 6 & 7 & 8 & 9 & 10 & 11 \\
\hline $\boldsymbol{Y}\left(y_{i}\right)$ & 2 & 3 & 14 & 5 & 16 & 5 & 17 & 7 & 8 & 9 & 10 \\
$\boldsymbol{C S}\left(\mathrm{mm}^{2}\right)$ & 50 & 50 & 95 & 50 & 95 & 50 & 300 & 185 & 95 & 50 & 50
\end{tabular}

overloading), so that if any of following penalty terms are flagged that solution is eliminated.

1) Cable crossing. In this study, both crossed edges (branches) have the chance to be selected individually. Before running the optimization process, the joint of every two-edges that may cross are detected and stored in a set. After that, during the optimization process, cable crossing can be detected in a solution, if any of those two-edges appears together.

2) Looping. A loop can be detected by comparing the total generated power of WTs and the summation of gathered power of all clusters into the OS.

3) Overloading. When the ampacity of a branch is higher than the admissible ampacity of the largest available

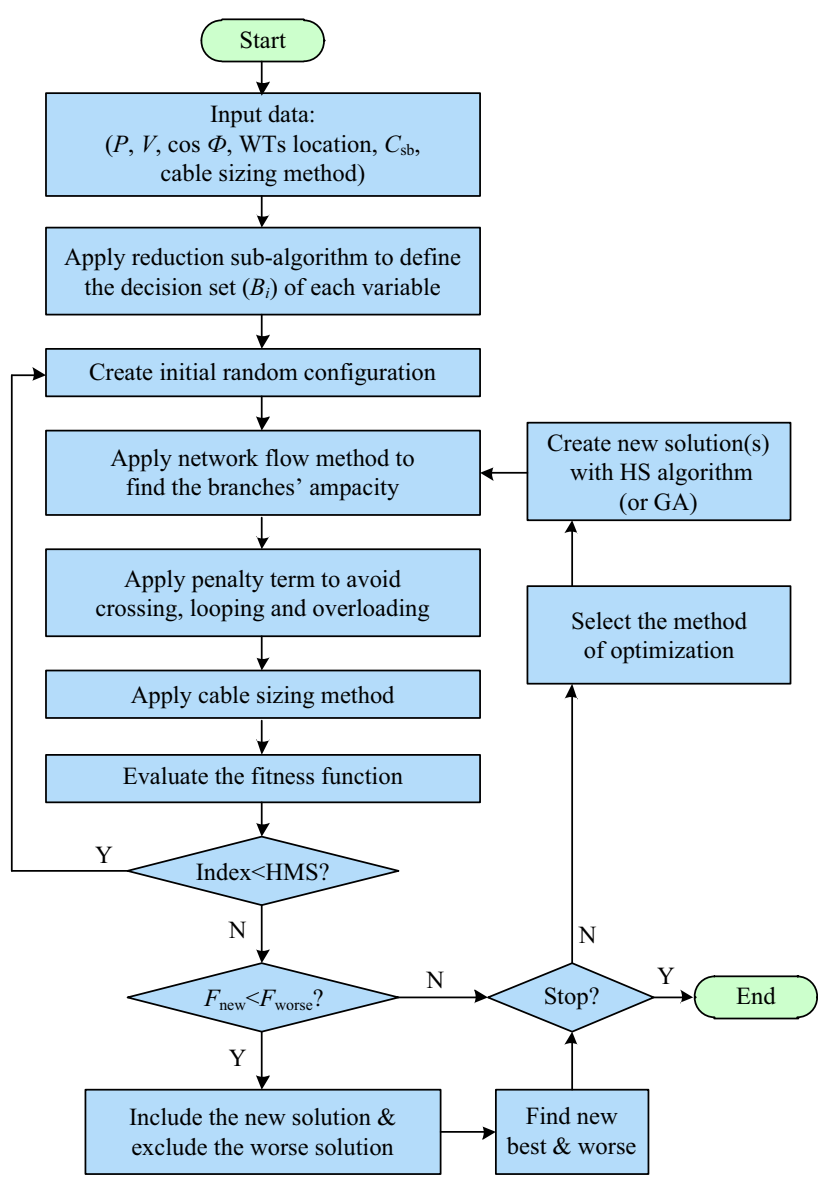

Fig. 2 Flowchart of optimization algorithm with HS (GA) 
dimension of subsea cables, overloading can easily be detected.

For the GA, a roulette wheel selection and $k_{i}$-based encoding method have been used, where $k_{i}$ is the number of choices for each individual optimization variable, which may not be a constant value. Therefore, both GA and HS algorithms are dealing with integer values and various numbers of choices for each individual optimization variable, according to the discrete decision set $\left(B_{i}\right)$. In order to improve the performance of GA multipoint crossover with random splice points has been considered, while mutation probability and population are assumed as 0.1 and $N$, respectively.

\subsection{Objective functions}

Our proposed simultaneous optimization consists of two terms for minimization of the trenching length and cable dimensions simultaneously. A constant $C_{\mathrm{sb}}$ is considered in this paper; however, an even variable $C_{\mathrm{sb}}\left(C_{\mathrm{sb}, i}=\right.$ $\left.\sum_{x=1}^{k_{i}} \beta_{x} C_{\mathrm{sb}, i, x}\right)$ could easily be applied to each optimization variable in the objective function, where $i$ is the optimization variable index, $x$ is the choice index of the decision set and $\beta_{x}$ a binary variable $[0,1]$ that is defined in (3).

The following objective function is formulated to minimize the CAPEX of electrical interconnection system of a given topology OWF.

$$
\left\{\begin{array}{l}
\min \sum_{i=1}^{N} D_{i, y_{i}}\left(C_{\mathrm{sb}}+\sum_{d=1}^{m} \lambda_{i}\left(a_{d}\right) \cdot C c_{i}\left(a_{d}\right)\right) \\
\text { s.t. } \sum_{d=1}^{m} \lambda_{i}\left(a_{d}\right)=1 \quad \forall \lambda_{i} \in\{0,1\}, a_{d} \in A
\end{array}\right.
$$

where $a_{d}$ is $d^{\text {th }}$ dimension of the cable set; $m$ is the number of available dimensions in the cable set; $N$ is the number of WTs (optimization variables); $y_{i}$ is the assigned value to $i^{\text {th }}$ WT as optimization variable (index of the next connected node); $C c_{i}\left(a_{d}\right)$ is the corresponding cost of assigned cable $\left(a_{d}\right)$ to $i^{\text {th }}$ turbine for each individual configuration; $C_{\mathrm{sb}}$ is the sum of shipping and burying costs; $D_{i, y_{i}}$ is the distance between $i^{\text {th }}$ WT and $y_{i}^{\text {th }}$, which can be calculated as follows:

$D_{i, y_{i}}=\sqrt{\operatorname{Re}\left(l o c_{i}-l o c_{y_{i}}\right)^{2}+\operatorname{Im}\left(l o c_{i}-l o c_{y_{i}}\right)^{2}}$

where $l o c_{i}$ and $l o c_{y_{i}}$ are the Cartesian location coordinates of $i^{\text {th }}$ WT and the next connected node index $\left(y_{i}^{\text {th }}\right)$, respectively.

In order to have an admissible total power loss, an inequality constraint has been added to the objective function as a penalty function:
$\Delta P_{\text {tot }}=\sum_{i=1}^{N} \Delta P_{i} \leq \Delta P_{\max }$

where $\Delta P_{\text {tot }}$ is the total power loss; $\Delta P_{\max }$ is the total maximum permitted active power loss; $\Delta P_{i}$ is the active power loss (13) of the connected branch to $i^{\text {th }} \mathrm{WT}$ in the worst case:

$\Delta P_{i}=3 R_{i}\left(a_{d}\right) I_{i, y_{i}}^{2} D_{i, y_{i}}$

where $R_{i}\left(a_{d}\right)$ is $\mathrm{AC}$ resistance of the assigned cable dimension $\left(a_{d}\right)$ connected to $i^{\text {th }} \mathrm{WT}$ at $90^{\circ} \mathrm{C}$.

According to (13) the power loss is proportional to the square of flowing current, thus in (14) the total cost of average power loss during a year is affected by square rate of the capacity factor (CF) in practice. In addition, due to smaller farm area of OWFs in comparison with its on-shore counterpart (identical power rating), the power loss of its electrical interconnection system is relatively lower (mostly, it is just about 1\%). Note that, according to the offshore wind characteristic, the annual capacity factors of installed OWFs are generally in the range of 0.33-0.54 $[25,26]$. Hence, in order to minimize the CAPEX for investors, the power loss is defined as a penalty function with its admissible value (lower than 2\%) [13]. Nevertheless, in case of obtaining identical fitness function among different optimal solutions the one with lower total power loss is selected. The annual cost of energy loss can represent the economic performance during the operational phase of the OWF over its lifetime, while maintenance cost is considered a constant value in all cases of this paper:

$C_{\Delta W}=8760 \cdot L C o E \cdot C F^{2} \cdot \sum_{i=1}^{N} \Delta P_{i}$

where $C_{\Delta W}$ is the annual cost of approximate energy loss; $C F$ is the capacity factor; $L C o E$ is the levelized cost of energy of OWF. In order to find the total cost of energy loss as an operational cost at the investment date, its net present value (NPV) during the OWF lifetime has been calculated [26, 27]. However, the annual profit of OWF is not considered, since it is the same in all cases. The aim is only to minimize some of the influencing terms of LCoE.

$N P V_{\Delta W}=\sum_{y=1}^{L T} \frac{C_{\Delta W}}{(1+r)^{y}}=\sum_{y=1}^{L T} \frac{8760 \cdot L C o E \cdot C F^{2} \cdot \Delta P_{t o t}}{(1+r)^{y}}$

where $N P V_{\Delta W}$ is the NPV term of the total cost of energy loss (without considering any $\mathrm{LCoE}$ reduction for OWF in the future); $r$ is the interest rate; $L T$ is the lifetime of the OWF. Therefore, the NPV term of power loss could easily be added to the objective function as a realistic term to find the overall cost of the electrical interconnection system. 
However, including this term into the objective function is not necessary. Because, the total power loss of OWFs is relatively low (about 1\%), and minimizing the CAPEX is a more significant issue for investors.

\section{Case study}

In this paper, a semi-symmetrical rectangular topology $(11 \times 6)$ consisting of 63 WTs is considered, where eleven turbines can harvest direct wind and WTs on the next arrays allocated with larger space particularly in order to minimize the overall wind wake effect. The WTs' spacing is assigned according to WT blades diameter $(D)$. Different admissible spacing has been defined in the literature, e.g. $3 D-7 D$ and $7 D-12 D[13,28-30]$. Thereby, the spacing of $5 D$ and $9 D$ are assumed between the WTs along an array $\left(d_{y}\right)$ and between the arrays $\left(d_{x}\right)$, respectively. Moreover, an intermediate single OS is assumed at the centroid of the OWF to decrease the interconnection CAPEX as well as the total power loss $[9,10,15,31]$. The OWF is assumed to be far from the shore, thus a high voltage transmission system is required for the connection of the OWF to the main grid, which is not investigated in this study. According to the available literature, the assumption values for optimization are given in Table 4 [27, 29, 32]. Two different Siemens WTs with 3.6 and 6.0 MW power rating have been assumed in this study while the diameters are 120 and $154 \mathrm{~m}$, respectively (SWT-3.6-120 and SWT-6.0154). The above-mentioned WTs employ a variable-speed doubly-fed induction generator (DFIG) and an individual step-up transformer with output voltage of $33 \mathrm{kV}$ at nacelle or pad-mount, according to the structure stamina of WTs. The DFIG manufacturers offer different characteristics options for reactive power generation [33]. Figure 3 shows the characteristic of active power $(P)$ versus reactive power $(Q)$ of a typical DFIG [34].

In order to consider the worst case in cable sizing (maximum ampacity), the power factor has been considered at 33\% leading or lagging reactive power, at fully rated active power $(\cos \phi=0.95)$. The injected current of each WT is calculated as:

$I_{G}=\frac{P_{G}}{\sqrt{3} V_{L} \cos \phi}$

where $P_{G}$ is the rated power of each WT; $V_{L}$ is the output line voltage of its internal transformer. A $690 \mathrm{~V} / 33 \mathrm{kV}$ step-up transformer is used to boost the voltage up to 33 $\mathrm{kV}$, since the stator output voltage of the WTs generator is typically $690 \mathrm{~V}$. The maximum permitted active power loss $\left(\Delta P_{\max }\right)$ of the electrical interconnection system is conventionally considered as $2 \%$ of the OWF capacity.

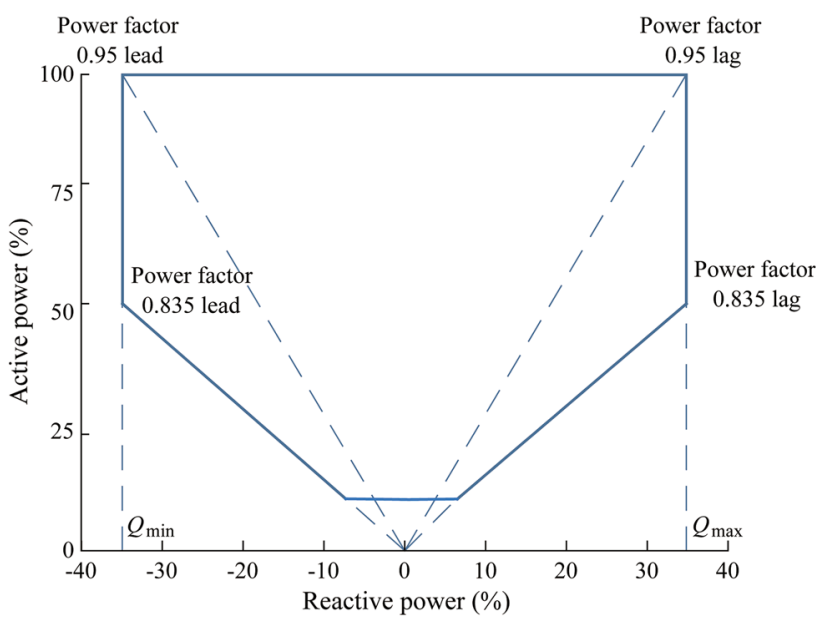

Fig. $3 P-Q$ characteristics of DFIG base WTs

All available dimensions of subsea (submarine) MV cables have been considered in the decision set of cable sizing problem, where $A=\{50,70,95,120,150,185,240$, $300,400,500,630\}$. According to shipping limitation or unavailability, a new set with a few choices can easily be replaced, thus it may even decrease $C_{\mathrm{sb}}$. The parameters of three-core copper conductor with XLPE insulator and armored subsea cable for $18-30 \mathrm{kV}(36 \mathrm{kV})$ are shown in Table $2[35,36]$. Note that, it represents only the dimensions that have been used in this paper.

The $C_{\mathrm{sb}}$ has significant contribution in the electrical interconnection CAPEX and may vary in different OWFs. A constant value has been assumed in this paper, conventionally; because, the outer diameter and weight of a subsea cable with maximum dimension of $630 \mathrm{~mm}^{2}$ is just about twice as thick and heavy as $50 \mathrm{~mm}^{2}$. However, variable $C_{\mathrm{sb}}$ $\left(C_{\mathrm{sb}, i, x}\right)$ can easily be applied to the objective function, if more practical detailed cost information about $C_{\mathrm{sb}}$ is provided.

\section{Results and discussions}

This simultaneous optimization is solved offline, relying on the HS algorithm as well as integer-based GA. Each algorithm is run 100 to 1000 times to frustrate the uncertainty of the metaheuristic algorithms and calculate their performances. In order to compare the performances of these two algorithms fairly, the stopping criteria of both algorithms set to individual maximum number of iteration so that only running times of the algorithms are relatively similar. This is because the number of generated offspring in any iteration of GA is much higher than in the HS algorithm (only one offspring). In order to evaluate the average performances of the GA and HS algorithms in different ranges of WTs, the optimal solutions of both 
Table 2 Submarine and subsea cable parameters

\begin{tabular}{|c|c|c|c|c|c|c|c|c|c|}
\hline MV cable $\left(a_{d}\right)\left(\mathrm{mm}^{2}\right)$ & 50 & 70 & 95 & 185 & 240 & 300 & 400 & 500 & 630 \\
\hline Ampacity in subsea (A) & 241 & 281 & 335 & 473 & 540 & 601 & 671 & 742 & 812 \\
\hline $\mathrm{AC}$ resistance at $90{ }^{\circ} \mathrm{C}(\Omega / \mathrm{km})$ & 0.493 & 0.342 & 0.247 & 0.127 & 0.1 & 0.08 & 0.063 & 0.05 & 0.041 \\
\hline Cost $(\$ / m)$ & 89 & 118 & 152 & 250 & 283 & 320 & 381 & 430 & 490 \\
\hline
\end{tabular}

cable sizing methods are calculated for various topologies of OWFs; e.g. $4 \times 5$ (with 18 WTs) and $4 \times 6$ (with 22 WTs). Table 3 indicates average estimations for the performance of various topologies and various cable sizing methods.

The results confirm the better performance of the HS algorithm to find the global optimal solution of this complex optimization problem, compared to the GA. This is because the new harmony (new solution) in the HS algorithm is improvised with a random selection for each optimization variable individually, by considering all existing harmonies (solutions) in HM. This feature increases the flexibility of the HS algorithm to produce better solutions in iterations compared to the GA as it was also demonstrated in the literature [20, 21, 23]. On the contrary, the GA only considers the two parent chromosomes (solutions) with single- or multi-point crossover. However, the GA simulation would be more compatible and perform well for partial cable sizing method, where multipoint crossover is considered; even its performance was relatively close to the HS algorithm or even better in some particular topologies. Because, in the partial cable sizing method, the cable dimensions of all branches in each route are the same and assigned only according to the ampacity of the last branch before joining to another route. On contrary, according to individual improvising of each optimization variable in the HS algorithm, it shows more compatibility with full cable sizing rather than partial cable sizing method. Higher performance and faster convergence of the HS algorithm compared to the GA is demonstrated together in Table 3 and Fig. 4 (fitness value versus iteration).

Additionally, the HS algorithm has found the optimal solution by considering all possible node connections (even non-feasible ones) in the decision set. Nevertheless, due to

Table 3 Comparison between performances of GA and HS

\begin{tabular}{lllll}
\hline Range of WTs & GA $(\%)$ & & \multicolumn{2}{l}{ HS algorithm $(\%)$} \\
\hline Cable sizing method & Full & Partial & Full & Partial \\
Range of 18-22 WTs & 84.9 & 91.5 & 97.6 & 94.8 \\
Range of 37-43 WTs & 73.3 & 84.6 & 91.3 & 88.6 \\
Range of 58-65 WTs & 57.1 & 72.4 & 83.7 & 76.8 \\
\hline
\end{tabular}

increased number of iterations to find global optimal solution of a larger problem, each optimization process takes much longer and obviously with lower performance. The fact that, full optimization yields identical results as the reduced optimization problem, verifies the functionality of the proposed reduction sub-algorithm.

In this section, the optimal electrical interconnection layout is comprehensively assessed. Variation in different influencing parameters and cable sizing methods are considered via three distinct scenarios to investigate their impact on the CAPEX and the optimal interconnection layout of a given OWF. Scenario I uses the full cable sizing method to find the simultaneous optimal interconnection configuration and cable sizing. Moreover, the impact of different $C_{\mathrm{sb}}$ and different WT power rating are investigated. Scenario II uses the partial cable sizing method to solve the similar simultaneous optimization. Scenario III employs a conventional radial configuration in order to compare and investigate different aspects.

The data the OWF considered for optimization is described in Table 4, where in the main cases power rating of $6 \mathrm{MW}$ and constant $C_{\mathrm{sb}}$ of $250 \$ / \mathrm{m}$ are considered to have a fair comparison among all the scenarios.

\subsection{Scenario I: full cable sizing method}

In this scenario, full cable sizing method is used for the simultaneous optimization. Two different comparisons are made to investigate the impact of various $C_{\mathrm{sb}}$ and power

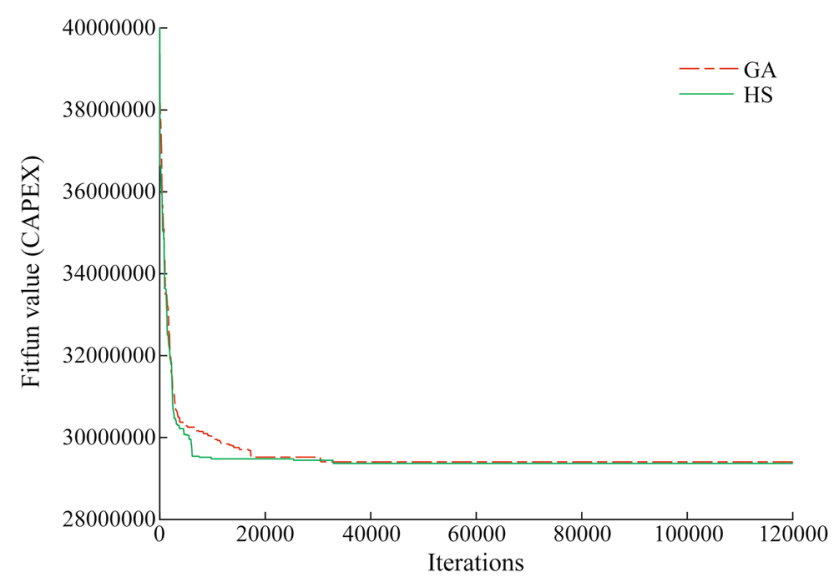

Fig. 4 Performance of HS and GA 
Table 4 Assumption for optimization

\begin{tabular}{ll}
\hline Parameter & Value \\
\hline No. of WTs & 63 \\
Topology & $n \times m: 6 \times 11$ \\
Spacing & $L_{x}=9 D, L_{y}=5 D$ \\
$C_{\text {sb }}$ (various in scenario I) & $250 \$ / \mathrm{m}, 500 \$ / \mathrm{m}$ \\
Rated power (various in scenario I) & $6 \mathrm{MW}$ \\
OWF capacity (various in scenario I) & $387 \mathrm{MW}$ \\
Capacity factor & 0.45 \\
OWF lifetime & 20 years \\
LoCE & $140 \$ / \mathrm{MWh}$ \\
Interest rate $(r)$ & $5 \%$ \\
Rated voltage & $33 \mathrm{~kW}$ \\
cos $\phi$ & 0.95 \\
\hline
\end{tabular}

ratings of WTs on the CAPEX and the optimal interconnection layout of a given OWF. Note that, any change in the space between the WTs along an array or between the arrays obviously changes the optimal interconnection layout. However, due to space limitations and pre-defined location of WTs in OWFs, this comparison is not investigated in this paper.

In the first comparison, the optimal electrical interconnection layouts of the identical OWF with the WT's power rating of $6 \mathrm{MW}$ are found in two cases with $C_{\mathrm{sb}}$ of 250 and $500 \$ / \mathrm{m}$ and shown in Fig. 5 and Fig. 6, respectively.

The results reveal that $100 \%$ increase in $C_{\mathrm{sb}}$ (from 250 $\$ / \mathrm{m}$ to $500 \$ / \mathrm{m}$ ) leads to $52 \%$ increase in the electrical interconnection CAPEX (from M\$29.352 to M\$ 44.656). However, the total trenching length is decreased by $3.7 \%$ $(2.318 \mathrm{~km})$ as $C_{\mathrm{sb}}$ affects about the length term of the simultaneous optimization formulation. The total active power loss in both optimal interconnection layouts (Fig. 5 and Fig. 6) are about $1 \%$, which is just half of the admissible loss in full capacity of the OWF.

In the third case of scenario I, the optimal electrical interconnection layouts of the identical OWF with $C_{\mathrm{sb}}$ of $250 \$ / \mathrm{m}$ are found, where WTs are considered with the power rating of 3.6 MW and the total capacity of 226.8 MW. Note that, the original diameter is $120 \mathrm{~m}$ instead of $154 \mathrm{~m}$, thus only per unit values can be compared. The optimal interconnection layout is shown in Fig. 7.

The results indicate that CAPEX of the interconnection system for both $6 \mathrm{MW}$ and $3.6 \mathrm{MW}$ are relatively similar (about $76 \$ / \mathrm{kW}$ ). In addition, the percentage of their total power losses is almost the same (about 1\%). However, the total trenching length is decreased, due to spacing reduction. It is also found that the optimal interconnection configuration of an OWF with lower power rating would be similar to the simple configuration of a traditional OWF, as the power rating of WTs in the last decade was in the range

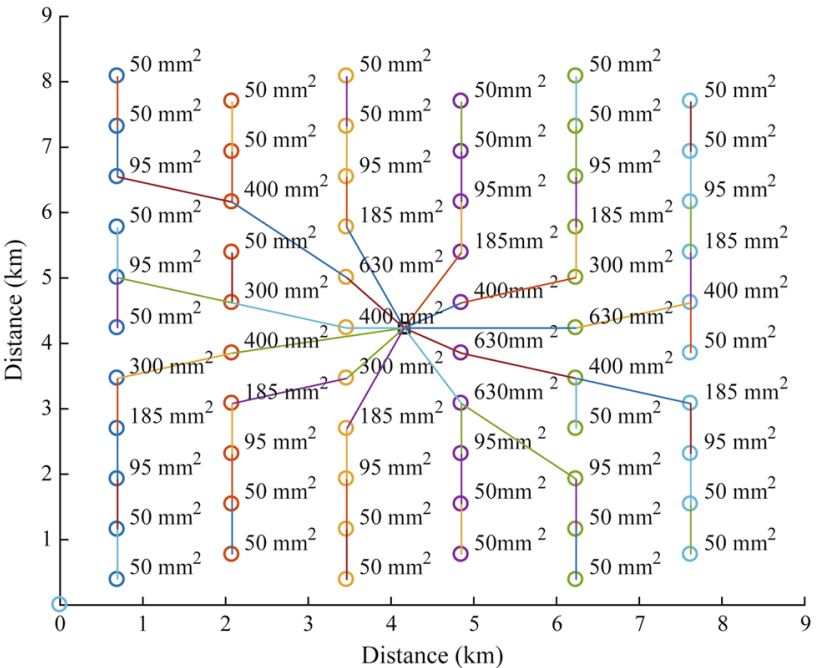

Fig. 5 Scenario I, optimal layout $\left(C_{\mathrm{sb}}\right.$ of $250 \$ / \mathrm{m}$ with $\left.6 \mathrm{MW}\right)$, CAPEX $=\mathrm{M} \$ 29.352 \&$ Losses $=4.039 \mathrm{MW} \& L_{\mathrm{T}}=62.740 \mathrm{~km}$

of 2-3.6 MW, and where a single low dimension cable could collect the generated power of several WTs.

\subsection{Scenario II: partial cable sizing method}

In this scenario, the partial cable sizing method is applied in the objective function so that only limited number of cable dimensions is considered to be available in the decision set $\left(50,185\right.$ and $\left.630 \mathrm{~mm}^{2}\right)$. In addition, cable dimensions of all branches on each route are identically assigned according to the ampacity of the last branch before joining another route or connecting to the OS. In partial cable sizing, shipping or burying might be easier and $C_{\mathrm{sb}}$ may decrease in practice, according to fewer changes in cable dimensions or using only a few cable dimensions. However, partial cable sizing may lead to over-dimensioning in some branches and increasing the 


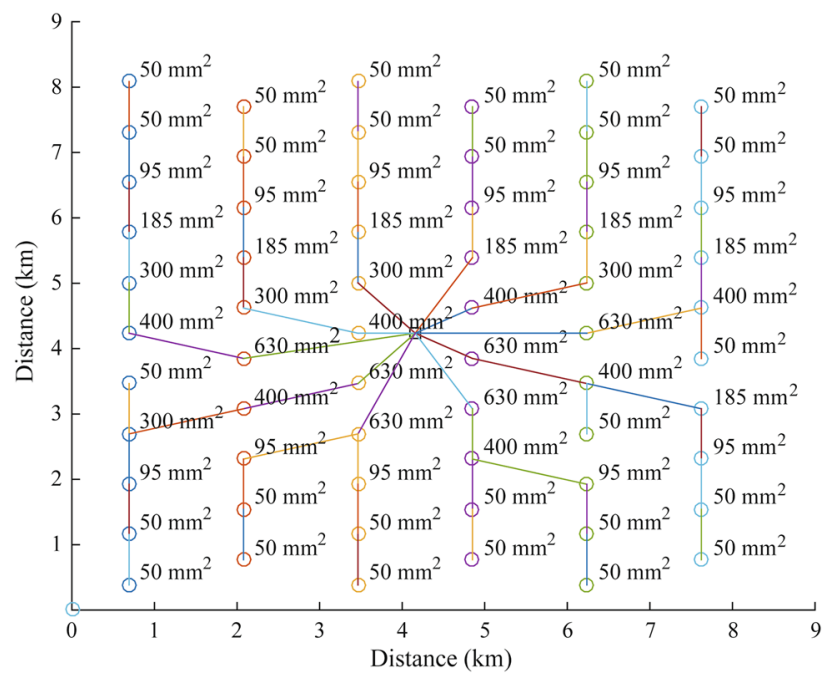

Fig. 6 Optimal layout $\left(C_{\mathrm{sb}}\right.$ of $500 \$ / \mathrm{m}$ with $\left.6 \mathrm{MW}\right), \mathrm{CAPEX}=\mathrm{M} \$$ $44.656 \&$ Losses $=3.927 \mathrm{MW} \& L_{\mathrm{T}}=60.421 \mathrm{~km}$

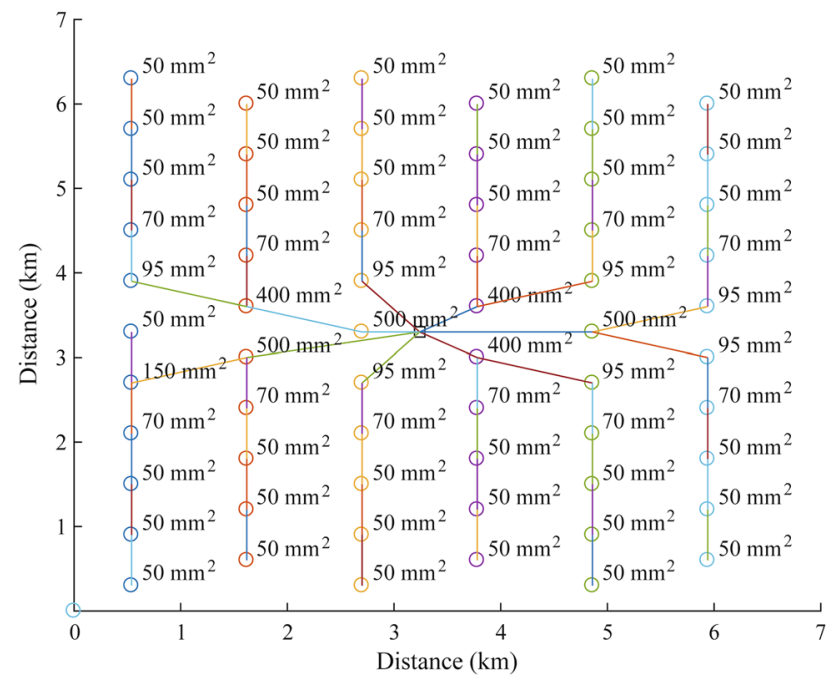

Fig. 7 Optimal layout $\left(C_{\mathrm{sb}}\right.$ of $250 \$ / \mathrm{m}$ with $\left.3.6 \mathrm{MW}\right)$, CAPEX= M\$ 17.686 and Losses $=2.3507 \mathrm{MW} \& L_{\mathrm{T}}=43.904 \mathrm{~km}$

CAPEX, which is investigated in this section. Fig. 8 shows the optimal interconnection layout of the identical OWF in Scenario II.

The optimal interconnection layout of this scenario can be compared with the main case in scenario I (Fig. 5). The investment and operational costs of the electrical interconnection systems, and the overall cost of all scenarios are indicated in Table 5. The NPV terms of the total cost of energy losses during the OWF lifetime are calculated as a variable operating cost in this paper, while maintenance cost is considered a constant value in all scenarios.

The results reveal that the CAPEX of interconnection system in scenario II is increased by $8.67 \%$ compared to scenario I. However, the overall costs increase will be only

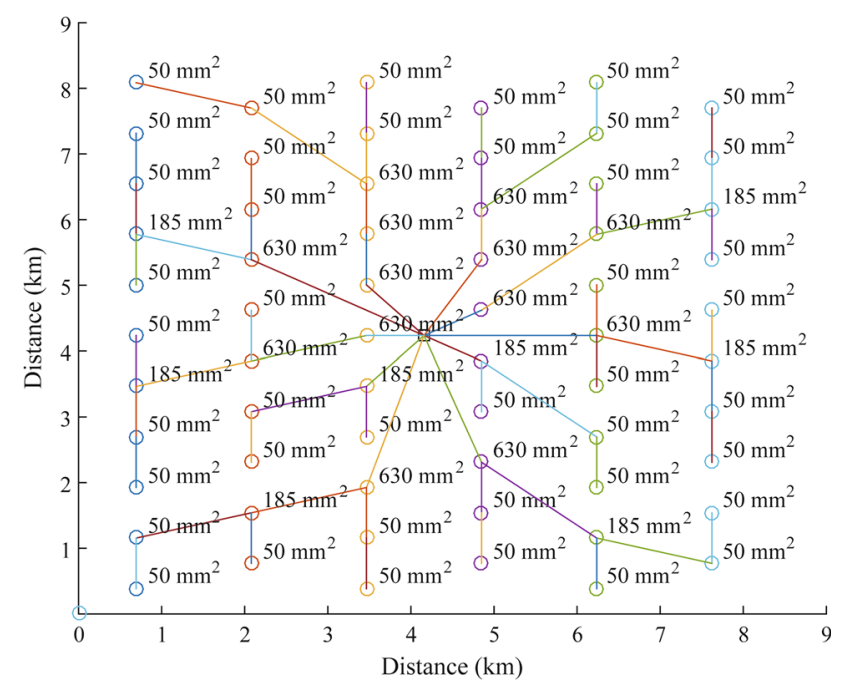

Fig. 8 Scenario II, optimal layout $\left(C_{\mathrm{sb}}\right.$ of $250 \$ / \mathrm{m}$ with $\left.6 \mathrm{MW}\right)$, $\mathrm{CAPEX}=\mathrm{M} \$ 31.897$ and Losses $=3.557 \mathrm{MW} \& L_{\mathrm{T}}=67.278 \mathrm{~km}$

by $2.5 \%$ (M 1.052), due to obtaining lower power loss, which is less than $0.92 \%$ ( $3.557 \mathrm{MW}$ ). Hence, scenario II also has good potential to be a justified solution, if partial cable sizing can provide just about $5.02 \% C_{\mathrm{sb}}$ reduction (if $C_{\mathrm{sb}} \approx 237 \$ / \mathrm{m}$ instead of $250 \$ / \mathrm{m}$ ) in practice. No details have been published about effective parameters in $C_{\mathrm{sb}}$, e.g., vessel capacity for various number dimensions or the length of subsea cables to ship in each journey to the OWF area. Note that, it is only an estimation according to the first comparison, since any change in $C_{\mathrm{sb}}$ for the new optimization process may change not only CAPEX but also the optimal layout.

\subsection{Scenario III: conventional radial layout}

Most of OWF projects in the last decade have used a typical conventional radial configuration[37]. In order to illustrate the significance of the proposed simultaneous optimization, the optimal interconnection layouts in scenarios I and II are additionally compared with a conventional radial layout, which is shown in Fig. 9 for the identical OWF while cable crossing is avoided.

The results of the typical radial electrical interconnection layout indicate that the CAPEX of interconnection system is increased to M\$38.755. Table 5 reveals that, according to the CAPEX, the optimal electrical interconnection of the scenarios I and II would save about $24.3 \%$ and $17.7 \%$, respectively. However, the total power loss of $0.69 \%(2.667 \mathrm{MW})$ in this scenario is relatively low as expected, due to the over-dimensioning of the cables away from the OS. Hence, the overall cost saving during the OWF lifetime will be M\$ 5.159 (about 10.97\%) and M\$ 4.107 (about $8.74 \%$ ), respectively. 
Table 5 Comparison between capital investment and operational cost of different scenarios $\left(C_{\mathrm{sb}}\right.$ of $250 \$ / \mathrm{m}$ with $\left.6 \mathrm{MW}\right)$

\begin{tabular}{lccc}
\hline Parameters & Scenario I & Scenario II & Scenario III \\
\hline CAPEX (investment cost) & 29.352 & 31.897 & 38.755 \\
Total power loss (387 MW capacity) (\%) & 1.04 & 0.92 & 0.69 \\
Cost of energy loss (operational cost as NPV) (M) & 12.501 & 11.008 & 8.257 \\
Overall costs of interconnection system (M) & 41.853 & 42.905 & 47.012 \\
\hline
\end{tabular}

\section{Conclusion}

This paper proposes an optimization formulation to minimize the CAPEX of the electrical interconnection system of OWFs, in order to help designers to reach the necessary balance between technical performance and economic costs. The simultaneous optimal electrical interconnection configuration and cable sizing of a given topology OWF is found. An HS algorithm as a high performance optimization algorithm for discrete problems is used to solve this complex and discrete optimization problem. The optimization results are compared with an integer-based GA as a proven optimization algorithm. The results demonstrate the higher performance of the HS algorithm in comparison with the GA. The proposed formulation can be easily adopted to on-shore wind farms as well. The main breakthrough of this study is to propose two individual options as optimal cable sizing methods (full and partial) in the objective function to assess the optimal interconnection layout of an identical OWF with a given topology comprehensively. A typical radial configuration is also compared with these two optimal layouts. Furthermore, two additional investigations reveal the significant influences of $C_{\mathrm{sb}}$ and WT's power rating over the CAPEX and their impact on the appearance of the optimal layout of interconnection system. The results illustrate that variation in the WT's power rating completely changes the optimal interconnection layout of the OWF. In addition, it turns out that increasing $C_{\mathrm{sb}}$ increases the CAPEX and often changes the optimal interconnection layout.

Comparative results in Table 5 indicate that, according to the CAPEX, both scenarios I and II as optimal solutions in this paper would save about $24.3 \%$ and $17.7 \%$, respectively, compared to scenario III. However, according to the overall cost during the OWF lifetime their saving will be $10.97 \%$ and $8.74 \%$, respectively. In conclusion, scenario I by applying full cable sizing method is the best solution in general, according to the applied cost assumptions. Note that, scenario II could also be a justified solution or even the best solution, if partial cable sizing method can provide

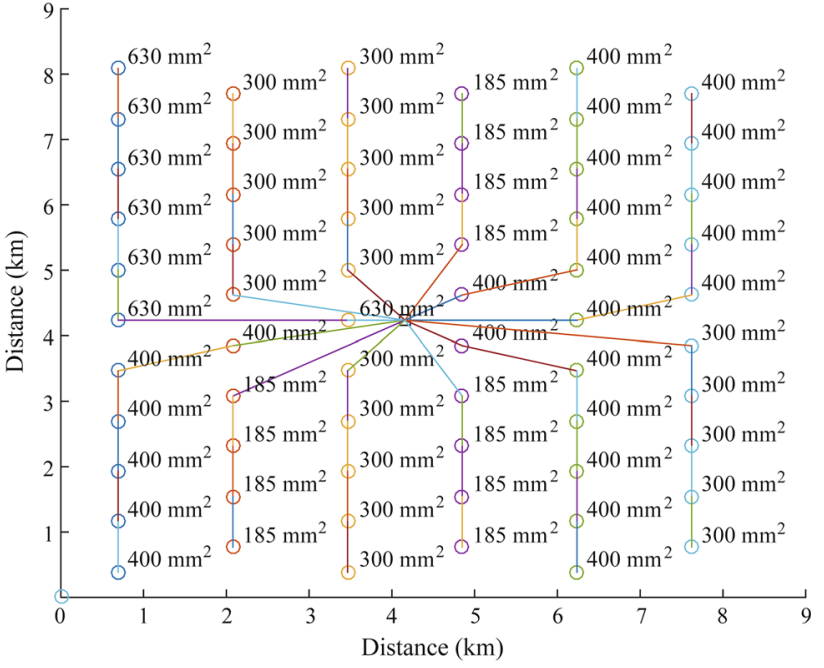

Fig. 9 Scenario III, optimal layout $\left(C_{\mathrm{sb}}\right.$ of $250 \$ / \mathrm{m}$ with $\left.6 \mathrm{MW}\right)$, CAPEX $=\mathrm{M} \$ 38.755 \&$ Losses $=2.667 \mathrm{MW} \& L_{\mathrm{T}}=63.163 \mathrm{~km}$

enough $C_{\mathrm{sb}}$ reduction in practice. Moreover, the total active power loss in full capacity of the OWF for both optimal scenarios turned out to be just half of the admissible value (about $1 \%$ ).

In the future work, the proposed methodology could be similarly developed to find the optimal connection between OWFs via a high voltage grid and linkage to the on-shore grid(s). Besides, the single- and multi-OS solutions as well as $66 \mathrm{kV}$ and $33 \mathrm{kV}$ interconnection systems will be investigated in real cases of OWFs.

Acknowledgements The technical support of Prof. István Erlich, chair of Electrical Power Systems department in Duisburg-Essen University, Duisburg, Germany is greatly appreciated.

Open Access This article is distributed under the terms of the Creative Commons Attribution 4.0 International License (http:// creativecommons.org/licenses/by/4.0/), which permits unrestricted use, distribution, and reproduction in any medium, provided you give appropriate credit to the original author(s) and the source, provide a link to the Creative Commons license, and indicate if changes were made. 


\section{References}

[1] EY (2015) Offshore wind in Europe. Ernst and Young Global Limited report $(\mathrm{EY})$

[2] Erlich I, Shewarega F, Feltes CW et al (2013) Off-shore wind power generation technologies. Proc IEEE 101(4):891-905

[3] Kamalakannan C, Suresh LP, Dash SS et al (2014) Power electronics and renewable energy systems. Proceedings of ICPERES, Springer, Germany

[4] Quinonez-Varela G, Ault GW, Lara OA et al (2007) Electrical collector system options for large offshore wind farms. IET Renew Power Gener 1(2):107-114

[5] Dutta S, Overbye TJ (2011) A clustering based wind farm collector system cable layout design. In: Proceedings of IEEE power and energy conference at Illinois (PECI), Champaign, USA, 25-26 Feb 2011, 6 pp

[6] Dutta S, Overbye TJ (2012) Optimal wind farm collector system topology design considering total trenching length. IEEE Trans Sustain Energy 3(3):339-348

[7] Shin JS, Kim WW, Kim JO (2011) Study on designing for inner grid of offshore wind farm. J Clean Energy Technol 3(4):265-269

[8] Chen Y, Dong Z (2013) A novel technique for the optimal design of offshore wind farm. J Mod Power Syst Clean Energy 1(3):258-263

[9] Banzo M, Ramos A (2011) Stochastic optimization model for electric power system planning of off-shore wind farms. IEEE Trans Power Syst 26(3):1338-1348

[10] Lumbreras S, Ramos A (2013) Optimal design of the electrical layout of an off-shore wind farm applying decomposition strategies. Power Syst 28(2):1434-1441

[11] Pillai A, Chick J, Johanning L et al (2015) Offshore wind farm electrical cable layout optimization. Eng Optim 47(12): $1689-1708$

[12] González J, Payán B, Santos J (2013) A new and efficient method for optimal design of large offshore wind power plants. IEEE Trans Power Syst 28(3):3075-3084

[13] Gonzalez-Longatt FM, Wall P, Regulski P et al (2012) Optimal electric network design for a large offshore wind farm based on a modified genetic algorithm approach. IEEE Syst $\mathrm{J}$ 6(1):164-172

[14] Dahmani O, Bourguet S, Machmoum M et al (2015) Optimization of the connection topology of an offshore wind farm network. IEEE Syst J 9(4):1519-1528

[15] Hou P, Hu W, Chen Z (2016) Optimisation for offshore wind farm cable connection layout using adaptive particle swarm optimisation minimum spanning tree method. IET Renew Power Gener 10(5):694-702

[16] Hou P, Hu W, Chen C et al (2017) Overall optimization for offshore wind farm electrical system. Wind Energy 20(6):1017-1032

[17] Hou P, Hu W, Soltani M et al (2017) Combined optimization for offshore wind turbine micro siting. Appl Energy 189:271-282

[18] Dan B, Pei-Jun Y, Li-Xum S (2007) Optimal design method of looped water distribution network. Syst Eng Theory Pract 27(7):137-143

[19] Chu CW, Lin MD, Liu GF et al (2008) Application of immune algorithms on solving minimum-cost problem of water distribution network. Math Comput Model 48:1888-1900

[20] Yun HY, Jeong SJ, Kim KS (2013) Advanced harmony search with ant colony optimization for solving the traveling salesman problem. J Appl Math 2:1-8

[21] Geem ZW, Kim JH, Loganathan GV (2001) A new heuristic optimization algorithm: harmony search. Simulation 76(2): 60-68
[22] Wang L, Xu Y, Mao Y et al (2010) A discrete harmony search algorithm. Commun Comput Inf Sci 98:37-43

[23] Geem ZW (2006) Optimal cost design of water distribution networks using harmony search. Eng Optim 38(3):259-280

[24] ENTSOE (2011) Offshore transmission technology report. ENTSOE (European Network of Transmission System Operators for Electricity)

[25] Cavazzi S, Dutton AG (2016) An offshore wind energy geographic information system (OWE-GIS) for assessment of the UK's offshore wind energy potential. Renew Energy 87:212-228

[26] Levitt AC, Kempton W, Smith AP et al (2011) Pricing offshore wind power. Energy Policy 39:6408-6421

[27] Voormolen JA, Junginger HM, Sark WV (2016) Unravelling historical cost developments of offshore wind energy in Europe. Energy Policy 88:435-444

[28] Pookpunt S, Ongsakul W (2016) Design of optimal wind farm configuration using a binary particle swarm optimization at Huasai district. Energy Convers Manag 108:160-180

[29] Siemens (2014) SCOE-society's costs of electricity: how society should find its optimal energy mix. Siemens, Germany

[30] Samorani M (2013) The wind farm layout optimization problem. Springer, Berlin, pp 21-38

[31] Sedighi M, Moradzadeh M, Kukrer O et al (2016) Optimal electrical interconnection configuration of off-shore wind farms. In: Proceedings of international conference on clean and green energy, Kuala Lumpur, Malaysia, 25-26 June 2016, 6 pp

[32] GWEC (2015) Global wind report 2015, annual market update. http://gwec.net/publications/global-wind-report-2/global-windreport-2015-annual-market-update/

[33] Erlich I, Wilich M, Feltes C (2007) Reactive power generation by DIFIG based wind farms with AC grid connection. In: Proceedings of European conference on power electronic and applications, Aalborg, Denmark, 2-5 Sept 2007, 10 pp

[34] Gashi A, Kabashi G, Ahmetaj S et al (2012) Simulation the wind grid code requirements for wind farms connection in Kosovo transmission grid. Energy Power Eng 4:482-495

[35] Cable O (2015) Ningbo orient wires and cables Co., Ltd. China Ningbo Subsea Cable Institute, Ningbo, China

[36] Nexans (2013) Submarine power cables. Nexans, Update June 10,2013

[37] Projet (2013) Projet de Parc Éolien au Large de Saint-Nazaire, P. du Banc de Guérande

Mohsen SEDIGHI received the Ph.D. degree (2017) from Eastern Mediterranean University (EMU), Gazi Magusa, North Cyprus, Turkey, and the M.Sc. degree (2007) from Semnan University, Semnan, Iran, both in Electrical Power Engineering. He is also a faculty member with the department of Electrical Engineering at Islamic Azad University (IAU), Sari branch, Iran. His research interests are power system design, renewable energy, distributed generation (DG) and optimization techniques applied to the complex planning problems in power systems.

Mohammad MORADZADEH is an Assistant Professor in Electrical Power Engineering in Department of Electrical Engineering at the Shahid Rajaee Teacher Training University, Lavizan, Tehran, Iran. He received his Ph.D. degree from Ghent University, Ghent, Belgium, in 2012, and the M.Sc. degree from K.N. Toosi University of Technology, Tehran, Iran, in 2007, both in Electrical Power Engineering. He worked as a Postdoctoral Fellow in Ghent University during 2013-2015, and then joined University of Windsor, Windsor, ON, Canada as a Program Development Administrator for an academic year. Lately, he served as a Senior Lecturer in the 
Department of Engineering and Technology at the University of Huddersfield, UK, during 2015-2017.

Osman KUKRER received the B.S., M.S., and Ph.D. degrees in electrical engineering from the Middle East Technical University (METU), Ankara, Turkey, in 1979, 1982, and 1987, respectively. From 1979 to 1985, he was a Research Assistant with the Department of Electrical and Electronics Engineering, METU. From 1985 to 1986, he was with the Department of Electrical and Electronics Engineering, Brunel University, London, U.K. He is currently a Professor with the Department of Electrical and Electronic Engineering, Eastern Mediterranean University, Gazi Magusa, Turkey. He has co-authored more than 50 technical papers in international journals and conferences. His research interests include power electronics, control systems, and signal processing. Dr. Kukrer is a senior member of the IEEE, and a member of the Chamber of Electrical Engineers in North Cyprus.
Murat FAHRIOGLU Obtained the B.S. (Hon) degree in Electrical Engineering from Michigan State University, in 1993, the M.S. degree (1994) and the Ph.D. degree (1999) from the University of Wisconsin-Madison. He is currently an Assoc. Professor at Middle East Technical University-Northern Cyprus Campus in the Department of Electrical and Electronics Engineering. His main research is electric power systems and more specifically in the design of demand management contracts between electric utilities and their customers to avoid forced outages. He is also working on renewable energy integration into power systems and assessment of solar and wind energy resources. His most recent research is about interconnecting the power systems of neighboring countries to enhance renewable energy use. 